\title{
Aeromonas bacteremia in an elderly immunocompetent patient
}

\author{
Paul Sebo $^{1}$, Ken Sakbani ${ }^{1}$, Peter Rohner ${ }^{2}$, and Gaetan Gavazzi ${ }^{1}$ \\ ${ }^{1}$ Department of Geriatrics, ${ }^{2}$ Laboratory of Microbiology, Geneva University Hospitals, Geneva, Switzerland
}

\begin{abstract}
We report the case of an elderly immunocompetent patient with Aeromonas hydrophila bacteremia without evidence of portal of entry. Despite several risk factors for a poor outcome, such as impaired renal function, two positive blood cultures, and community-acquired infections, the patient survived. Antimicrobial susceptibility was normal. Unknown polycystic liver disease was discovered and misdiagnosed as a hepatic abscess at the time of the bacteremia which was confirmed by repeated CT scans. Because of the absence of other risk factors for Aeromonas bacteremia, hepatic polycystic disease may take part in the onset of Aeromonas sp bacteremia as well as immunosenescence.
\end{abstract}

(Aging Clin Exp Res 2006; 18: 344-346)

${ }^{\circ}$ 2006, Editrice Kurtis

\section{INTRODUCTION}

Aeromonas species, previously classified as Vibrionaceae, are Aeromonadaceae ubiquitous gram-negative bacilli, widely distributed in aquatic ecosystems (freshwater and marine environments) and isolated with increased frequency in summer. In humans, Aeromonas causes a wide variety of infections, including wound infections, bacteremia and diarrhoea, in immunocompromised patients and rarely in immunocompetent individuals (1).

We report a case of bacteremia caused by Aeromonas hydrophila in an immunocompetent patient without evident exposure to an aquatic source in a geriatric hospital in Geneva in 2001.

\section{CASE REPORT}

An 87-year-old man was admitted to the Geriatric Hospital of Geneva in July 2001 with fever and chills which had lasted two days before admittance, and fatigue during the previous month. His medical history included
Parkinson's disease since 1995, a sylvian stroke with regressive aphasia in 1999, hypertension, and chronic renal insufficiency.

At admission, physical examination revealed a relatively healthy man, with a normal body temperature and no other significant symptoms.

Laboratory tests showed pancytopenia $(\mathrm{Hb} 12.5 \mathrm{~g} / \mathrm{dl}$, WBC count 3.3x10p9/1, platelet count $119000 / \mathrm{mm} 3$ ). Electrolyte levels were within normal range, serum creatinine concentration was $145 \mathrm{umol} / \mathrm{l}$, and Protein C reactive $169 \mathrm{mg} / \mathrm{l}$.

Two blood cultures revealed Aeromonas hydrophila. The isolated germ was susceptible to second- and thirdgeneration cephalosporin and quinolones, and resistent to amoxicillin, co-amoxyclav and first-generation cephalosporin. Urine and stool cultures were negative. The chest film was normal. An abdominal scan showed multiple hypodense lesions, the largest being $18 \mathrm{~mm}$ in cross section. At this point, differential diagnosis indicated metastatic septic lesions or hepatic cysts. In accordance with susceptibility patterns, the patient was treated with ciprofloxacin $500 \mathrm{mg}$ 2x/day for one month. Clinical evolution was favorable over the first five days. Pancytopenia disappeared on the 6 th day. PCR was less than $3 \mathrm{mg} / \mathrm{l}$ after 3 weeks. As there was no change in the hepatic lesions after 1 and 3 months, our diagnosis was polycystic liver disease. Exposure to Aeromonas may have been by drinking freshwater near his home but no bacteriological analysis was performed.

\section{DISCUSSION}

Aeromonas species are considered as predominantly pathogenic to poikilotherms (1). However, they have emerged as an important human pathogen and the cause of a wide range of diseases. Transmission may occur through water and food $(2,3)$.

Key words: Aeromonas, bacteremia, elderly, immunocompetent, polycystic hepatic disease.

Correspondence: G. Gavazzi, MD, PhD, Département de Médecine Gériatrique et Communautaire, Centre Hospitalier Universitaire de Grenoble, 38043 Grenoble cedex 09, France.

E-mail: Ggavazzi@chu-grenoble.fr

Received March 25, 2005; accepted in revised form August 11, 2005. 
Aeromonas are responsible for the following infections: mild to severe wound infections, almost always following traumatic aquatic exposure or after immersion in water immediately after burns $(3,4)$; and acute or chronic diarrhoea in the community, with increased isolation in patients with traveller's diarrhoea, but also in long-term care settings $(5,6)$. Aeromonas $s p$ causes bacteremia mostly in patients with malignancy or other immunocompromising conditions such as serious liver disease (mostly hepatic cirrhosis), or traumatic injuries (7).

The largest trial on Aeromonas infections was carried out in Taiwan from 1989 to 1998, with 104 episodes of monomicrobial Aeromonas bacteremia (7). Interestingly, there was a predominance of males (80\%), and age ranged from 14 to 96 , with a mean of 53.6 years. The two common underlying diseases were hepatic cirrhosis $(54 \%)$ and malignancies (21\%). Most cases were community-acquired (74\%). Impaired renal function at presentation, more than one set of positive blood cultures, community-acquired infections, hepatic cirrhosis as underlying illness, and secondary bacteremia, were independently associated with a fatal outcome. Gender, age and bacterial species did not affect the clinical outcome. An additional infection focus was found in only $43 \%$ of patients. Despite the age of the patient reported here, who presented with several worsened prognostic factors (impaired renal function, two positive blood cultures, community-acquired infections), his condition improved rapidly and the outcome was favorable.

Janda and Abbott proposed four major categories of persons susceptible to Aeromonas septicemia (8). Two are immunocompromised adults (due to malignant diseases or hepatic cirrhosis) and infants under 2 years of age with multiple underlying medical complications, both of which develop sepsis by bacterial translocation from the intestine into the bloodstream after colonization of the gastrointestinal tract. A third group is observed to develop sepsis as a result of wound infections. A fourth group, an extremely small collection of cases, is composed of patients with neither underlying defects nor exposure to sources of freshwater.

Our case was of interest because of the presence of polycystic liver disease, never reported before, as a possible favoring condition and the absence of exposure to a freshwater source. Infection of cysts occurs essentially by enterobacteria, and there are no reports of Aeromonas as causal agent (9). In our case however, it is not clear whether there was a cyst infection. Abdominal scanning revealed no argument for infection, there was no change after antibiotic treatment, and percutaneous aspiration of cysts to confirm infection was not carried out because of the favorable evolution. In addition, the decreased immune function related to aging may have contributed to the onset of bacteremia. However, there are not many reports on Aeromonas infections in the elderly. One might argue that very old individuals are probably rarely exposed to the bacterium, but also that even scarce exposure is sufficient to induce infection. This may also have been the case for our patient, because of exposure to freshwater. According to the patient, "drinking freshwater near his home" was a possibility, but when was not ascertained. In addition, this hypothesis could not be confirmed by serial bacteriological analysis of the freshwater in question. It should be mentioned that this is the first Aeromonas bacteremia report in Switzerland. In the University Hospital of Geneva there have only been nine episodes of Aeromonas bacteraemia in the last ten years (5 Aeromonas hydrophila, 3 caviae, 1 sobria) (unpublished data, Dr Rohner P.). The incidence of disease caused by Aeromonas in Switzerland is not well known. In the only trial conducted among adults with diarrhoea in Switzerland, stool specimens from throughout the country showed $8 \%$ Aeromonas sp, but there is no information regarding the locations of other infections and bacteremia (10).

Aeromonas sp. are naturally resistent to penicillin, ampicillin and ticarcillin, but susceptible to second- and third-generation cephalosporin, and other antibiotics such as quinolones, aminoglycosides, cotimoxazole, chloramphenicol and tetracyclines. There is a clear difference in antibiotic susceptibility among species, underlining the need for identification and susceptibility testing of all isolates (1). In our case, the isolated Aeromonas hydrophila was susceptible to second- and third-generation cephalosporin and quinolones as known for Aeromonas hydrophila.

Cases of Aeromonas bacteremia are still rare in presumably immunocompetent individuals. However, such infections may become more frequent in healthy elderly as far as the elderly population increases and may be more frequently exposed to Aeromonas sp bacteria.

\section{REFERENCES}

1. Janda JM, Abbott SL. Evolving concepts regarding the genus Aeromonas: an expanding panorama of species, disease presentations, and unanswered questions. Clin Infect Dis 1998; 27: 332-44.

2. Buchanan RL, Palumbo SA. Aeromonas hydrophila and Aeromonas sobria as potential food poisoning species: a review. J Food Safety 1985; 7: 15-29.

3. Gold WL, Salit IE. Aeromonas hydrophila infections of skin and soft-tissue: report of 11 cases and review. Clin Infect Dis 1993; 16: 69-74.

4. Kienzle N, Muller M, Pegg S. Aeromonas wound infection in burns. Burns 2000; 26: 478-82.

5. Yamada S, Matsushita S, Dejsirilert S, Kudoh Y. Incidence and clinical symptoms of Aeromonas-associated travellers' diarrhoea in Tokyo. Epidemiol Infect 1997; 119: 121-6.

6. Bloom HG, Bottone EJ. Aeromonas hydrophila diarrhoea in a long-term care setting. J Am Geriatr Soc 1990; 38: 804-6.

7. Ko WC, Lee HC, Chuang YC, Liu CC, Wu JJ. Clinical features 
and therapeutic implications of 104 episodes of monomicrobial Aeromonas bacteraemia. J Infect 2000; 40: 267-73.

8. Janda JM, Guthertz LS, Kokka RP, Shimada T. Aeromonas species in septicemia: laboratory characteristics and clinical observations. Clin Infect Dis 1994; 19: 77-83.

9. Rosenfeld L, Bonny C, Kallita M, et al. Les polykystoses hépa- tiques: principales complications et prise en charge. Gastroenterol Clin Biol 2002; 26: 1097-106.

10. Rohrbach M, Gualzata MD, Hollfelder R, Viollier AF, Viollier M, Viollier EH. Which are the most frequently occurring enteropathogenic bacteria in clinical practice? Schweiz Med Wochenschr 1988; 118: 1850-3. 